\title{
Estudio multicéntrico, de seroprevalencia de IgG Toxoplasma gondii en niños con cáncer de la red del Programa Infantil Nacional de Drogas Antineoplásicas en la Región Metropolitana, Chile
}

\author{
Toxoplasma gondii IgG seroprevalence in children with cancer from National Children's \\ Antineoplastic Drug Program network in the Metropolitan Region, Chile: a multicenter \\ study
}

Marcela Zúñiga Contreras', Isabel Noemi Hauck², Romina Valenzuela Cortés ${ }^{3}$, Marlon Barraza Olivares ${ }^{4}$ y María Elena Santolaya de Pablo 5

${ }^{1}$ Becada Infectología Pediátrica. Universidad de Chile. Santiago, Chile.

${ }^{2}$ Hospital Luis Calvo Mackenna. Departamento de Pediatría y Cirugía Infantil Oriente. Universidad de Chile. Santiago, Chile.

${ }^{3}$ Unidad de Investigación. Departamento de Pediatría y Cirugía Infantil Oriente. Universidad de Chile. Santiago, Chile.

${ }^{4}$ Unidad de Farmacia. Hospital Luis Calvo Mackenna. Santiago, Chile.

${ }^{5}$ Hospital Luis Calvo Mackenna. Departamento de Pediatría y Cirugía Infantil Oriente. Universidad de Chile. Santiago, Chile.

Declaramos no tener conflicto de interés.

Este proyecto se financió con fondos del proyecto FONDECYT No 1161662

Recibido: 28 de septiembre de 2020 / Aceptado: 19 de febrero de 202

\section{Resumen}

Introducción: Toxoplasma gondii es un protozoo que afecta a un tercio de la población mundial y cuya seroprevalencia actualizada en niños con cáncer en nuestro medio, se desconoce. Objetivo: Describir la seroprevalencia de IgG anti- $T$. gondii en población pediátrica con cáncer atendida en hospitales públicos de la Región Metropolitana, Chile. Método: Estudio transversal de pacientes pediátricos oncológicos atendidos en la ciudad de Santiago. Se tomó una muestra de suero de 100 pacientes entre junio de 2016 y mayo de 2019. Se les realizó ELISA IgG T. gondii. Resultados: Se analizaron 100 muestras, 51\% de sexo masculino. Resultaron 12 positivas, 84 negativas y 4 indeterminadas. Los sueros de los pacientes se estratificaron por edad: $0-5$ años $43 \%$, 6-10 años 29\%, 11-15 años 20\% y > 15 años, $8 \%$. El $61 \%$ eran pacientes con leucemia aguda. El porcentaje de mujeres con IgG positiva fue de $21 \%$ en comparación a $4 \%$ en hombres $(\mathrm{P}<0,0011)$. Conclusión: El 84\% de los niños en tratamiento por cáncer son seronegativos para $T$. gondii, por lo que es importante educar en la prevención de la adquisición de este parásito en esta población, por el riesgo de desarrollar enfermedad grave con riesgo de muerte.

Palabras clave: toxoplasmosis; cáncer; seroprevalencia.

\section{Abstract}

Background: Toxoplasma gondii is a protozoan that affects a third of the world population and whose updated seroprevalence in children with cancer in our environment is unknown. Aim: To describe the seroprevalence of IgG anti-T. gondii in pediatric population with cancer treated in hospitals of the Metropolitan Region, Chile. Method: Crosssectional study of the population of pediatric cancer patients treated in Santiago city, A serum sample was taken from 100 patients between June 2016 and May 2019. ELISA IgG T. gondii was performed. Results: Of 100 children, $51 \%$ male. 12 were positive (12\%), 84 negative ( $84 \%)$ and 4 indeterminate (4\%). The stratification by age showed $43 \%$ patients between $0-5$ years, $29 \%$ between $6-10$ years, $20 \%$ in the group of $11-15$ years and $8 \%$ in patients $>15$ years. Sixty one percent of the samples came from patients with acute leukemia. The percentage of women who tested positive for IgG was $21 \%$ compared to $4 \%$ in men $(\mathrm{P}<0.0011)$. Conclusion: $84 \%$ of children undergoing cancer treatment are seronegative for $T$. gondii, so it is important to educate on the prevention of the acquisition of this parasite in this population, due to the risk of developing serious and fatal disease.

Keywords: toxoplasmosis; cancer; seroprevalence.

\section{Correspondencia a:}

María Elena Santolaya de Pablo

msantola@uchile.cl 
seroprevalencia de $\operatorname{IgG} T$. gondii en niños con leucemia aguda de $36,6 \%{ }^{4}$.

Siendo desconocida la prevalencia actual de $\operatorname{IgG} T$. gondii en nuestro medio -la única medición realizada en el país data de más de 20 años-, se diseñó el presente estudio, cuyo objetivo fue describir la seroprevalencia de IgG anti- $T$. gondii en niños con cáncer atendidos en la Región Metropolitana (RM) de Chile entre junio de 2016 y mayo de 2019.

\section{Objetivo}

Describir la seroprevalencia de IgG anti-Toxoplasma gondii en población pediátrica con cáncer atendidos en hospitales públicos de la RM.

En condiciones inmunológicas normales, la infección por $T$. gondii es usualmente asintomática y autolimitada ${ }^{4}$, pese a que se ha descrito su asociación con el desarrollo de diversas enfermedades tales como cirrosis hepática, epilepsia, esquizofrenia ${ }^{4,5}$ e incluso leucemia en niños ${ }^{4}$.

Sin embargo, en hospederos inmunocomprometidos es una enfermedad oportunista, que adquiere relevancia y gravedad. En estos casos, puede producirse por primoinfección o, generalmente, por reactivación de una infección latente ${ }^{6}$, que podría afectar gravemente al sistema nervioso central (SNC), miocardio u órganos componentes del sistema retículo endotelial, gatillando una enfermedad diseminada que amenaza con conducir a la muerte ${ }^{1}$.

Los pacientes con cáncer son un grupo considerable de hospederos inmunocomprometidos, tomando en cuenta que cada año se diagnostican cerca de 300.000 casos de cáncer en personas bajo 19 años en el mundo ${ }^{4}$. La neoplasia más frecuente es la hematológica, que representa cerca de $45 \%$ del total de nuevos diagnósticos pediátricos. En Chile, según el Registro Nacional de Cáncer Infantil, se diagnostican cada año aproximadamente 500 nuevos casos de neoplasias en niños, de los cuales $38 \%$ corresponden, a leucemia, $19 \%$ a tumores del SNC y $11 \%$ a linfoma ${ }^{7}$.

La mayoría de las series de seroprevalencia de toxoplasmosis en pacientes inmunocomprometidos han sido descritas en pacientes con síndrome de inmunodeficiencia adquirida, receptores de trasplante de órganos sólidos o de precursores hematopoyéticos, personas que reciben inmunoterapia, o bien, aquellos que sufren de enfermedades reumatológicas y mesenquimopatías de la niñez y reciben diferentes tratamientos inmunosupresores ${ }^{1,2,8}$. La seroprevalencia de toxoplasmosis en pacientes pediátricos con quimioterapia antineoplásica ha sido poco evaluada ${ }^{8}$. En un estudio realizado en el Hospital Dr. Luis Calvo Mackenna, de Santiago de Chile, en el año 1991, se objetivó una seroprevalencia para IgG $T$. gondii en niños con cáncer en quimioterapia de $37,5 \%{ }^{9}$. En el ámbito internacional, un estudio casocontrol realizado en Irán entre 2016-2017, encontró una

\section{Materiales y Métodos}

\section{Diseño global del estudio}

Se realizó un estudio descriptivo, transversal, en 120 muestras de suero congeladas a $-70^{\circ} \mathrm{C}$ previamente obtenidas en el contexto del proyecto FONDECYT $\mathrm{N}^{\circ} 1161662$ entre junio de 2016 y mayo de 2019, provenientes de pacientes pediátricos con cáncer cursando episodios de neutropenia febril y atendidos en los seis hospitales públicos de la red del Programa Infantil Nacional de Drogas Antineoplásicas (PINDA) de la RM, a saber: Hospital de Niños Dr. Luis Calvo Mackenna, Hospital de Niños Roberto del Río, Hospital de Niños Dr. Exequiel González Cortés, Complejo Asistencial Dr. Sótero del Río, Hospital Clínico San Borja Arriarán y Hospital San Juan de Dios. Las muestras se seleccionaron mediante muestreo aleatorio desde el bio-repositorio antes descrito. Se excluyeron las muestras de pacientes receptores de trasplante de precursores hematopoyéticos. Se incluyó sólo una muestra por paciente, recopilando de ellos información de datos demográficos (edad, sexo, nacionalidad, región de origen, hospital de origen) y tipo de cáncer, para caracterizar a la población.

El estudio fue aprobado por el Comité de Ética para la investigación en seres humanos de la Facultad de Medicina de la Universidad de Chile.

\section{Determinación de IgG anti T. gondii}

Las concentraciones de anticuerpos IgG anti- $T$. gondii fueron medidas mediante técnica de enzimoinmunoanálisis de absorción (ELISA) (Euroimmun $\mathrm{AG}^{\circledR}$, Germany), de acuerdo con las especificaciones del fabricante. Las concentraciones de corte utilizadas para esta técnica fueron: menos de $0,9 \mathrm{UI} / \mathrm{mL}=$ negativo; entre 0,9 y $1,0 \mathrm{UI} / \mathrm{mL}=$ no concluyente; y mayor a 1,0 $\mathrm{UI} / \mathrm{mL}=$ positivo. 


\section{Análisis estadístico}

Se calculó la prevalencia de IgG positiva para $T$. gondii y se determinó el respectivo intervalo de confianza al $95 \%$ utilizando el programa Epidat versión 3.0 versión 3.1 2006. La muestra fue estratificada por edad y se estimó la proporción de serología positiva en cada estrato. Las variables categóricas se presentaron como frecuencias y porcentajes. Se definió como variable dependiente la seroprevalencia de $\operatorname{IgG} T$. gondii y se evaluó su asociación con las variables explicativas (edad, sexo, nacionalidad,

Tabla 1. Seroprevalencia de lgG T. gondii. Características socio-demográficas y clínicas de pacientes con cáncer atendidos en 6 hospitales del PINDA de la Región Metropolitana

\begin{tabular}{lc}
\hline Variable & \% del total $(\mathbf{n}=\mathbf{1 0 0})$ \\
Sexo & 51 \\
Masculino & 49 \\
Femenino & \\
\hline Edad (años) & 43 \\
$0-5$ & 29 \\
$6-10$ & 20 \\
$11-15$ & 8 \\
$>15$ & \\
\hline
\end{tabular}

Región de origen de pacientes chilenos $(n=97)$

$\begin{array}{lc}\text { Metropolitana } & 63 \\ \text { II-IV Región } & 17 \\ \text { VI-X Región } & 15 \\ \text { Sin dato } & 2\end{array}$

Hospital

Hospital Roberto del Río

Hospital Luis Calvo Mackenna

Hospital Exequiel González Cortés

Hospital Sótero del Río

Hospital San Juan de Dios

Hospital San Borja Arriarán

63

17

15

2

\section{Patología de base}

Leucemia linfoblástica aguda

Leucemia no linfoblástica aguda

Recaída de leucemia

12

9

8

Tumor sólido

Linfoma

Recaída de tumor sólido

Otros hospital de origen, región de origen, tipo de cáncer). Para el análisis de asociación se utilizaron los test de $\chi^{2}$ o de Fischer. Los resultados indeterminados no fueron utilizados en el análisis. Se consideró significativo un valor de $\mathrm{P}<0,05$. Los análisis estadísticos se realizaron usando el programa estadístico R studio versión 1.2.5033 2009-2019.

\section{Resultados}

De un total de 120 muestras, se analizaron 100, tras la exclusión de 20 muestras repetidas o insuficientes. Las 100 muestras correspondieron a 100 pacientes: $43 \%$ entre 0 y 5 años, 29\% entre 6 y 10 años, 20\% entre 11 y 15 años y $8 \%$ mayor de 15 años. El 51\% fue de sexo masculino. Las características socio-demográficas y clínicas son descritas en la Tabla 1. El 97\% de los niños incluidos en la muestra fueron chilenos y $62,5 \%$ residentes en la RM. En relación con el tipo de cáncer, $81 \%$ tenía un cáncer hematológico, $15 \%$ un tumor sólido y $4 \%$ otro tipo de cáncer.

De los 100 sueros analizados, $12 \%$ resultaron positivos para la determinación de anticuerpos IgG anti-T. gondii (IC 95\%, 5,1-18,9), 84\% negativos y 4\% indeterminados (Figura 1). La proporción de mujeres con IgG anti- $T$. gondii positivo fue mayor que la proporción de hombres seropositivos ( 21 vs. $4 \%, \mathrm{P}=0,011)$. La prevalencia de seropositividad entre los distintos centros mostró diferencias estadísticamente significativas $(\mathrm{P}=0,04)$ (Tabla 2).

No se encontraron diferencias al comparar la prevalencia entre grupos etarios, región de origen ni patología de base (Tabla 2).

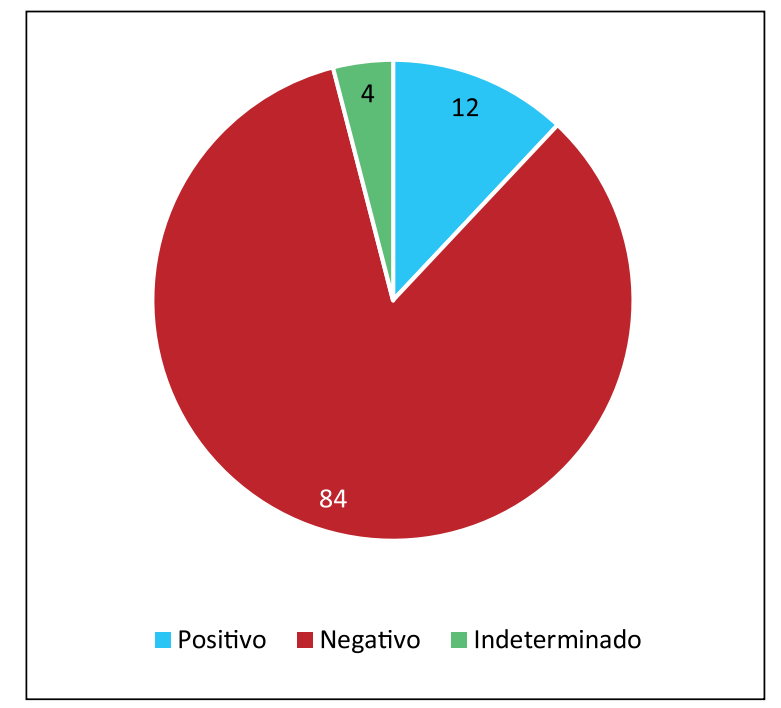

Figura 1. Resultado de IgG anti-Toxoplasma gondii. 


\begin{tabular}{|c|c|c|c|}
\hline Variable & $\begin{array}{l}\% \text { IgG Toxoplasma gondii positivo } \\
\text { (proporción) } n=12\end{array}$ & $\begin{array}{l}\text { \% IgG Toxoplasma gondii negativo } \\
\text { (proporción) } n=84\end{array}$ & Valor $p$ \\
\hline Sexo & & & 0,011 \\
\hline Femenino & $21(10 / 47)$ & $79(37 / 47)$ & \\
\hline Masculino & $4(2 / 49)$ & $96(47 / 49)$ & \\
\hline Edad (años) & & & 0,64 \\
\hline $0-5$ & $17(7 / 41)$ & $83(34 / 41)$ & \\
\hline $6-10$ & $7 \quad(2 / 29)$ & $93(27 / 29)$ & \\
\hline $11-15$ & $11(2 / 18)$ & $89(16 / 18)$ & \\
\hline$>15$ & $12(1 / 8)$ & $88 \quad(7 / 8)$ & \\
\hline Región & & & 0,76 \\
\hline Metropolitana & $15(9 / 60)$ & $85(51 / 60)$ & \\
\hline II-IV Región & $6(1 / 17)$ & $94(16 / 17)$ & \\
\hline VI - X Región & $7(1 / 15)$ & $93(14 / 15)$ & \\
\hline Extranjeros & $0 \quad(0 / 3)$ & $100 \quad(3 / 3)$ & \\
\hline Sin dato & $0 \quad(0 / 2)$ & $100 \quad(2 / 2)$ & \\
\hline Hospital & & & 0,04 \\
\hline Hospital Roberto del Río & $12(3 / 26)$ & $89(23 / 26)$ & \\
\hline Hospital Luis Calvo Mackenna & $4(1 / 23)$ & $96(22 / 23)$ & \\
\hline Hospital Exequiel González Cortés & $0 \quad(0 / 19)$ & $100(19 / 19)$ & \\
\hline Hospital Sótero del Río & $25(3 / 12)$ & $75(9 / 12)$ & \\
\hline Hospital San Juan de Dios & $38 \quad(3 / 8)$ & $63 \quad(5 / 8)$ & \\
\hline Hospital San Borja Arriarán & $25 \quad(2 / 8)$ & $75 \quad(6 / 8)$ & \\
\hline Patología de base & & & 0,12 \\
\hline Leucemia linfoblástica aguda & $10(3 / 30)$ & $90(27 / 30)$ & \\
\hline Leucemia no linfoblástica aguda & $14(4 / 28)$ & $86(24 / 28)$ & \\
\hline Linfoma & $50(1 / 2)$ & $50(1 / 2)$ & \\
\hline Recaída de leucemia & $12(2 / 17)$ & $88(15 / 17)$ & \\
\hline Tumor sólido & $7 \quad(1 / 15)$ & $93(14 / 15)$ & \\
\hline Otros & $25 \quad(1 / 4)$ & $75 \quad(3 / 4)$ & \\
\hline
\end{tabular}

\section{Discusión}

La seroprevalencia de $\operatorname{IgG} T$. gondii en niños con cáncer en tratamiento quimioterápico atendidos en centros PINDA de la RM fue de 12\% (IC 95\%, 5,1-18,9), resultado inferior a lo previamente reportado en la publicación de un estudio caso-control en niños con cáncer hematológico $(36,6 \%)^{4}$, otro estudio caso-control en niños con linfoma $(19,1 \%)^{10}$, un meta-análisis en adultos con cáncer $(20 \%)^{11}$, y un estudio caso control que comprobó una prevalencia de $66,7 \%$ de serología positiva en adultos con cáncer en quimioterapia $^{12}$. Además, pese a que no existen estudios actuales de seroprevalencia global en Chile, nuestros resultados son concordantes con lo encontrado por Barnafi y cols. ${ }^{13}$ donde la seroprevalencia bajo los 19 años fue en promedio de $12 \%$.

Nuestros hallazgos en niños con cáncer se pueden explicar por varios factores: un descenso de la prevalencia global de toxoplasmosis en nuestro país ${ }^{13}$ por un lento pero progresivo cambio de hábitos higiénicos y alimentarios de nuestra población, la mejoría del saneamiento ambiental, una mayor educación para la salud y una 
mayor escolaridad o, debido a que durante el tratamiento quimioterápico se ha objetivado una baja no sólo de los neutrófilos, sino también de las inmunoglobulinas, en especial $\operatorname{IgM}$ e $\operatorname{IgG}^{14,15}$. Existen, por otra parte, estudios avalando la preservación de la sensibilidad y especificidad del test de ELISA para IgG anti $T$. gondii cuando las determinaciones de IgG se realizan en muestras conservadas en bio-repositorios ${ }^{16}$, particularmente si las muestras han sido conservadas en alícuotas y descongeladas sólo una vez, como fue en el caso de nuestro estudio.

Este trabajo constata que, pese a que casi la mitad de las muestras correspondieron a mujeres, la proporción de mujeres positivas para $\operatorname{IgG} T$. gondii fue significativamente mayor que la encontrada en los hombres, hallazgo concordante con otros estudios en adultos con cáncer ${ }^{12,16,17}$ y distinto a lo encontrado en un estudio en Estados Unidos de América realizado entre 2009 y 2010 , con una prevalencia mayor en hombres $(p<0,05)^{18}$. Estas variaciones de prevalencia entre países podrían deberse a diferencias socio-culturales y de costumbres, tales como el cuidado de las mascotas, particularmente los gatos, el cuidado del jardín, la manipulación de carne cruda o parcialmente cocida, limpieza de frutas y verduras, entre otras actividades domésticas ${ }^{12,17}$.

En cuanto a la edad, no hubo diferencia significativa entre grupos etarios. Esto es similar a lo descrito en un estudio de casos y controles de serología para $T$. gondii en niños con patología hematológica maligna ${ }^{4}$, lo que podría relacionarse con una adquisición de la infección a temprana edad, sin aumento a medida que pasan los años.

En relación a la comparación de prevalencia entre los centros donde fueron atendidos los niños, hubo una diferencia estadísticamente significativa $(p<0,05)$, lo que podría deberse a que cada centro recibe pacientes de distintas regiones de Chile y, con ello, con diferencia de costumbres y hábitos. Sería interesante realizar estudios posteriores para conocer cuáles son las prácticas alimenticias, si viven en zonas urbanas o rurales y su relación con mascotas.

Como limitación de este estudio está el análisis retrospectivo de los datos. Sería importante realizar estudios prospectivos de seroprevalencia de toxoplasmosis en pacientes pediátricos con cáncer de todo el país en el momento del debut de la patología oncológica, con datos epidemiológicos precisos como el consumo de carne cruda o insuficientemente cocida, área de vivienda (rural o urbana), disponibilidad de agua potable, alcantarillado, tratamiento de aguas servidas, contacto con gatos y sus heces.

\section{Conclusiones}

Este estudio mostró que la frecuencia de infección medida a través de la determinación IgG $T$. gondii positivo fue de $12 \%$, cifra inferior a la descrita anteriormente. Nuestro trabajo aporta nueva información y establece la necesidad de estudiar la seroprevalencia al diagnóstico de los pacientes con el cuadro oncológico y antes del inicio de quimioterapia para prevenir las complicaciones graves de la toxoplasmosis que pueden llegar a ser mortales ${ }^{1,6}$. Estos resultados instalan una base para mayores estudios futuros en pediatría tanto en población oncológica como sana.

\section{Referencias bibliográficas}

1.- Ikebe T, Sasaki H, Takata H, Miyazaki Y, Ohtsuka E, Saburi Y, et al. Toxoplasmic encephalitis with untreated hairy cell leukemia variant. Intern Med. 2016; 55 (21): 3175-80. doi: 10.2169/internalmedicine.55.6668.

2.- Hamid. D. Prevalence of toxoplasmosis among cancer patients. Int J Adv Res. 2017; 5 (7): 1362-6. doi: 10.21474/IJAR01/4846.

3.- Shin D W, Cha D Y, Hua Q J, Cha G H, Lee Y H. Seroprevalence of Toxoplasma gondii infection and characteristics of seropositive patients in general hospitals in Daejeon, Korea. Korean J Parasitol. 2009; 47 (2): 125-30. doi: 10.3347/kjp.2009.47.2.125.

4.- Kalantari N, Rezanejad J, Tamadoni A, Ghaffari S, Alipour J, Bayani M. Association between Toxoplasma gondii exposure and paediatrics haematological malignancies: A case-control study. Epidemiol Infect. 2018; 146 (15): 1896902. doi: $10.1017 / \mathrm{S} 0950268818002194$.

5.- $\quad$ Torrey E F, Bartko J J, Yolken R H. Toxoplasma gondii and other risk factors for schizophrenia: An update. Schizophr Bull. 2012; 38 (3): 642-7. doi: $10.1093 / \mathrm{schbul} / \mathrm{sbs} 043$.

6.- Ruskin J, Remington J S. Toxoplasmosis in the compromised host. Ann Intern Med. 1976; 84 (2): 193-9. doi: 10.7326/0003-4819-84-2-193.

7.- Departamento de Epidemiología. Ministerio de Salud. Primer informe del Registro Nacional de Cáncer Infantil de Chile (menores de 15 años), RENCI. Quinquenio 2007-2011. Primera edición. Santiago de Chile 2018. http:// www.ipsuss.cl/ipsuss/site/artic/20180117/ asocfile/20180117150429/informe renci_2007_2011registro_nacional_c_ncer infantildepto_epidemiolog_aminsal2018.pdf.

8.- Remington J S, Israelski D M. Toxoplasmosis in patients with cancer. Clin Infect Dis. 1993; 17 (Suppl 2): S423-35. doi: 10.1093/clinids/17. supplement_2.s423.

9.- Gottlieb B, Noemi I, Reyes H, Volpi C, Cerva $\mathrm{J} \mathrm{L}$, Jorquera H, et al. Toxoplasmosis en niños con patologías oncológicas: estudio prospectivo de 200 casos. Parasitol Día.1991 jul-dic. 15
(3/4): 117-21. http://bases.bireme.br/cgi-bin/ wxislind.exe/iah/online/?IsisScript=iah/iah.xis $\& \mathrm{src}=$ google \&base $=$ LILACS\&lang $=\mathrm{p} \&$ nextA ction=lnk\&exprSearch=109462\&indexSearch $=$ ID.

10.- Duan Y, Zhi Y, Liu Y, Zhou N, Li F, Hao X, et al. Toxoplasma gondii infection in children with lymphoma in Eastern China: seroprevalence, risk factors and case-control studies. Epidemiol Infect. 2019; 147: e305. doi: 10.1017/ S0950268819001869.

11.- Jiang C, Li Z, Chen P, Chen L. The seroprevalence of Toxoplasma gondii in Chinese population with cancer a systematic review and meta-analysis. Med (United States). 2015; 94 (50): 1-7. doi: 10.1097/ MD.0000000000002274.

12.- Ali M I, Abd E 1 Wahab W M, Hamdy D A, Hassan A. Toxoplasma gondii in cancer patients receiving chemotherapy: seroprevalence and interferon gamma level. J Parasit Dis [Internet]. 2019; 43 (3): 464-71. Available from: https:// doi.org/10.1007/s12639-019-01111-9. 
Immunopathol (Madr) [Internet]. 2003; 31 (6): 303-10. Available from: http://dx.doi. org/10.1016/S0301-0546(03)79203-9.

15.- Perkins J L, Harris A, Pozos T C. Immune dysfunction after completion of childhood leukemia therapy. J Pediatr Hematol Oncol. 2017; 39 (1): 1-5. doi:10.1097/ MPH.0000000000000697.

16.- Dard C, Bailly S, Drouet T, Fricker-Hidalgo H, Brenier-Pinchart M P, Pelloux H. Long-term sera storage does not significantly modify the interpretation of toxoplasmosis serologies. $\mathrm{J}$ Microbiol Methods [Internet]. 2017; 134: 3845. Available from: http://dx.doi.org/10.1016/j. mimet.2017.01.003

17.- Anvari D, Sharif M, Sarvi S, Aghayan S A, Gholami S, Pagheh A S, et al. Seroprevalence of Toxoplasma gondii infection in cancer patients: A systematic review and metaanalysis. Microb Pathog [Internet]. 2019; 129(November 2018): 30-42. Available from: https://doi.org/10.1016/j.micpath.2019.01.040.

18.- Mostafa N E S, Hamed E F A, Rahed H E S, Mohamed S Y, Abdelgawad M, Elasbali A M. The relationship between toxoplasmosis and different types of human tumors. J Infect Dev Ctries. 2018; 12 (2): 137-41. doi: 10.3855/ jidc. 9672 . 